Geometry $\&$ Topology

Volume 8 (2004) 735-742

Published: 17 May 2004

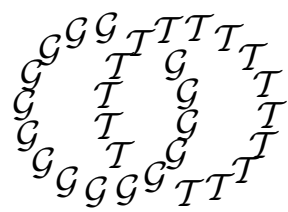

\title{
Computations of the Ozsváth-Szabó knot concordance invariant
}

\author{
Charles Livingston \\ Department of Mathematics, Indiana University \\ Bloomington, IN 47405, USA \\ Email: livingst@indiana.edu
}

\begin{abstract}
Ozsváth and Szabó have defined a knot concordance invariant $\tau$ that bounds the 4-ball genus of a knot. Here we discuss shortcuts to its computation. We include examples of Alexander polynomial one knots for which the invariant is nontrivial, including all iterated untwisted positive doubles of knots with nonnegative Thurston-Bennequin number, such as the trefoil, and explicit computations for several 10 crossing knots. We also note that a new proof of the Slice-Bennequin Inequality quickly follows from these techniques.
\end{abstract}

\section{AMS Classification numbers Primary: 57M27}

Secondary: 57M25, 57Q60

Keywords: Concordance, knot genus, Slice-Bennequin Inequality

Proposed: Peter Ozsváth

Seconded: Joan Birman, Ronald Fintushel
Received: 20 Febrary 2004

Accepted: 29 April 2004 
Using their theory of knot Floer homology, Ozsváth and Szabó [7] defined an invariant $\tau$ of knots in $S^{3}$ and showed that it induces a homomorphism $\tau: \mathcal{C} \rightarrow$ $\mathbf{Z}$, where $\mathcal{C}$ is the concordance group of smooth knots in $S^{3}$. Computations of $\tau$ for particular knots, and more generally the application of knot Floer homology to bound the 4-ball genus of knots (eg [6, 8, 9, 10]), depend upon a detailed understanding of its definition. Here we show that the most basic properties of $\tau$ developed in [7] are sufficient to yield its quick evaluation for a number of interesting examples including some pretzel knots of Alexander polynomial one, iterated untwisted doubles of knots with nonnegative Thurston-Bennequin number and some interesting 10 crossing knots.

Although we do not use the deeper theoretical work of Rudolph (eg [12]) here, in ways our approach parallels his extension of the results of KronheimerMrowka 4] on torus knots to more general knots and his proof of the SliceBennequin Inequality.

Three essential properties of $\tau$ are stated in the following theorem.

Theorem 1 There exists an integer valued knot invariant $\tau$ satisfying:

(1) $\tau(K \# J)=\tau(K)+\tau(J)$ and $\tau(-K)=-\tau(K)$ for all knots $K$ and $J$.

(2) The value of $\tau$ is bounded by the smooth 4-ball genus, $\tau(K) \leq g_{4}(K)$.

(3) For the $(p, q)$-torus knot with $p, q>0, T_{p, q}, \tau$ equals the 3 -sphere genus, $g_{3}\left(T_{p, q}\right)$. Specifically, $\tau\left(T_{p, q}\right)=(p-1)(q-1) / 2$.

An immediate consequence, as described in [7], is:

Corollary $2 \tau$ induces a homomorphism $\tau: \mathcal{C} \rightarrow \mathbf{Z}$ and $|\tau(K)| \leq g_{4}(K)$.

Proof That $|\tau(K)| \leq g_{4}(K)$ follows from $\tau(-K)=-\tau(K), g_{4}(K)=g_{4}(-K)$ and $\tau(K) \leq g_{4}(K)$. Next, if $K$ is concordant to $J$, then $K \#-J$ is slice, and hence of 4-genus 0. Thus, $\tau(K)+\tau(-J)=0, \tau(K)=\tau(J)$, and so $\tau$ is a concordance invariant.

The following appears in [7] as a corollary of the general relationship between $\tau(K)$ and the genus of surfaces bounded by $K$ in negative definite 4 -manifolds. Here we note that it follows immediately from Theorem [1.

Corollary 3 If $K_{+}$and $K_{-}$differ by a single crossing change, from positive to negative, then $0 \leq \tau\left(K_{+}\right)-\tau\left(K_{-}\right) \leq 1$. 
Proof The crossing change provides a genus 1 cobordism from $K_{+}$to $K_{-}$. Thus, $g_{4}\left(K_{+} \#-K_{-}\right) \leq 1$ and so $\left|\tau\left(K_{+}\right)-\tau\left(K_{-}\right)\right| \leq 1$. A negative crossing change converts $-T_{2,3}$ into the unknot, so $\left(K_{+} \#-T_{2,3}\right) \#-K_{-}$bounds a disk in $B^{4}$ with two double points of opposite signs. Tubing these double points together shows that $g_{4}\left(\left(K_{+} \#-T_{2,3}\right) \#-K_{-}\right) \leq 1$. Thus, $\left|\tau\left(K_{+}\right)-1-\tau\left(K_{-}\right)\right|$ $\leq 1$. Combining the two inequalities gives the desired result.

\section{Subsurfaces of Torus Knot Fibers}

For a surface $F$ we let $g(F)$ denote the genus of $F$. Recall that for any torus knot $T_{p, q}$ the complement is fibered over $S^{1}$ and the fiber $F$ realizes the 3 -genus of $T_{p, q},(p-1)(q-1) / 2$.

Theorem 4 Suppose that a knot $K$ is embedded in the interior of a fiber surface $F$ of a torus knot $T=T_{p, q}$ with $p q>0$ and that $K$ is null homologous on $F$, bounding a surface $G \subset F$. Then $\tau(K)=g_{4}(K)=g_{3}(K)=g(G)$.

Proof A Morse function $h: F-\operatorname{int}(G) \rightarrow[0,1]$ taking value 0 on $K$ and 1 on $T$ gives the cobordism (id $\times h): F-\operatorname{int}(G) \rightarrow S^{3} \times[0,1]$ from $T_{p, q}$ to $K$ of genus $g(F)-g(G)$. Hence, $T \#-K$ bounds a surface of genus $g(F)-g(G)$ in $B^{4}$ and $g_{4}(T \#-K) \leq g(F)-g(G)$. Thus $\tau(T)-\tau(K) \leq g(F)-g(G)$. By Theorem 1 $\tau(T)=g(F)$ and hence $g(G) \leq \tau(K)$. We then have the string of inequalities

$$
\tau(K) \leq g_{4}(K) \leq g_{3}(K) \leq g(G) \leq \tau(K)
$$

and these yield the desired result.

In the language of Rudolph (eg [12]), such surfaces $G$ on fibers of torus knots

are called quasipositive surfaces. One quick consequence of Rudolph's work is the following.

Corollary 5 The untwisted positive double of the trefoil and the pretzel knot $P(3,-5,-7)$ (both of Alexander polynomial one) have $\tau=1$.

Proof Rudolph [12] has drawn an explicit illustration of $P(3,-5,-7)$ on the fiber surface for the torus link $T_{5,5}$ and that illustration applies as well for $T_{5,6}$. Rudolph also indicates how a similar illustration can be drawn for the double of the trefoil. (Our sign convention for pretzel knots here is the opposite of that in [12] and is consistent with Rudolph's more general work on pretzel knots 
in 14]. The positive double is the double formed from two parallel unlinked copies of the knot by adding a clasp with two crossing points, both of which have positive sign.)

Corollary 6 The subgroup $\mathcal{P} \subset \mathcal{C}$ generated by knots of Alexander polynomial one contains a summand isomorphic to $\mathbf{Z}$. The knot $P(3,-5,-7)$ represents a generator of such a summand and in particular is not divisible: $P(3,-5,-7) \neq a K \in \mathcal{C}$ for any $a \neq \pm 1$.

Proof The argument is the same as in [5] where $\mathcal{A}$ instead of $\mathcal{P}$ is considered. Since $\tau$ maps $\mathcal{P}$ onto the free abelian group $\mathbf{Z}$ the map splits. Since $\tau(P(3,-5,-7))=1, P(3,-5,-7)$ cannot be divisible.

Rudolph's results along these lines have further applications. In particular, his work on pretzel knots [13] implies that the pretzel knot $P\left(t_{1}, \ldots, t_{k}\right)$ with all $t_{i}$ and $k$ odd (and its standard Seifert surface) embeds on the fiber of a torus knot with $p q>0$ if and only if $t_{i}+t_{j}<0$ for all $1 \leq i<j \leq k$ and thus for such pretzel knots $\tau=(k-1) / 2$.

Corollary 7 If $\hat{\beta}$ is the closure of a positive braid of $n$ strands and word length $k$, then $\tau(\hat{\beta})=\frac{k-n+1}{2}=g_{4}(\hat{\beta})$.

Proof The torus knot $T_{n, q}$ can be drawn as an $n$-stranded positive braid. Its fibered Seifert surface is formed from $n$ parallel disks joined by $q(n-1)$ twisted bands, one for each crossing point. Similarly, the Seifert surface $G$ for $\hat{\beta}$ is built from $n$ parallel disks by joining them with $k$ twisted bands. The resulting surface has Euler characteristic $n-k$, and hence genus $\frac{k-n+1}{2}$. By adding more bands to this surface, one can construct the fiber Seifert surface $F$ of the torus knot $T_{n, q}$ for some large $q$. Thus, removing a small open tubular neighborhood, on $G$, of the boundary of $G$ yields a surface homeomorphic to $G$ with boundary isotopic to $K$ in the interior of $F$. The proof is now completed by applying Theorem 4

\section{Examples}

In 33 there is a table listing the 4-genera of prime knots with 10 or fewer crossings, as then known. Since the appearance of that table, most of the unknown values have been determined. (See [15] for an updated table, where it 
appears that $10_{51}$ remains as the only unknown case.) However, the unknown cases of [3] continue to provide interesting test cases for new techniques, since classical methods could not resolve them. The few examples presented here were chosen because of their appearance in [6, 7], where direct application of the theory therein developed was used. Explicit calculations were not included in [7, in that they were lengthy and demanded the use of Mathematica.

Example 8 Using the notation of Rolfsen [11, the knots $10_{139}$ and $-10_{152}$ as shown in Figure 1 each are closures of positive braids with 3 strands and word length 10. Thus, by Corollary 7 the value of $\tau$ and $g_{4}$ for each of these is 4 . (The 4-ball genus of each of these was first computed in [2].)
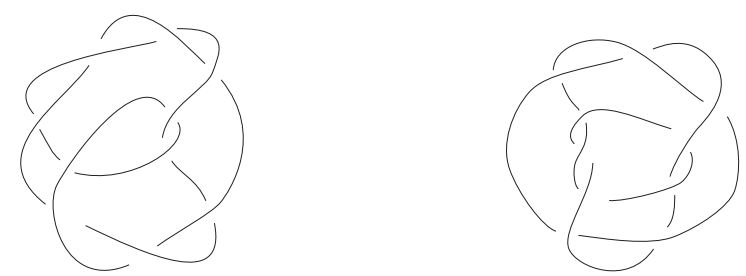

Figure 1: $10_{139}$ and $-10_{152}$

Example 9 The knot $-10_{161}$ as illustrated in Figure 2 is a 3 stranded braid with 9 positive and 1 negative crossings. Thus changing one crossing yields a knot with $\tau=4$. This implies that $\tau\left(-10_{161}\right) \geq 3$. But, since $g_{3}\left(-10_{161}\right)=3$, we also have that $\tau\left(-10_{161}\right) \leq 3$. So, $\tau\left(-10_{161}\right)=3$. Since $g_{3}\left(-10_{161}\right)=3$ it follows that $g_{4}\left(10_{161}\right)=3$ also. (The first calculation of the 4 -genus of this knot appeared in [16.)

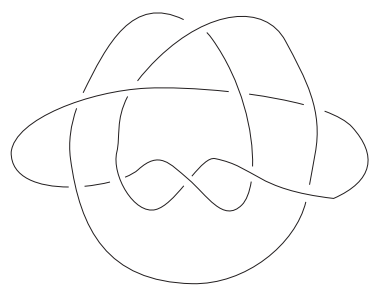

Figure 2: $-10_{161}$

Example 10 The knot $10_{145}$ is discussed in [6]. Changing orientation, $-10_{145}$ can be drawn as a braid with 4 strands and 11 crossings, 9 of which are positive. This is illustrated in Figure 3. Changing two crossing yield a knot $K$ which 
by Corollary 7 has $\tau(K)=4$ and so $\tau\left(-10_{145}\right) \geq 2$. On the other hand, $-10_{145}$ can be unknotted with 2 crossing changes, so $\tau\left(-10_{145}\right) \leq 2$. Hence, $\tau\left(-10_{145}\right)=2$. Since the unknotting number of $10_{145}$ is at most 2 , it follows that $g_{4}\left(10_{145}\right)=2$. (The first calculation of the 4 -genus of this knot also appeared in [16].)

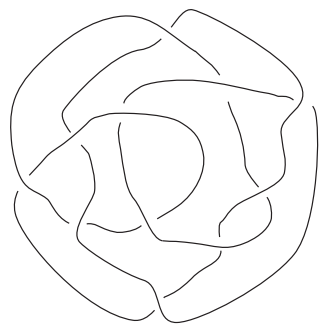

Figure 3: $10_{145}$

Examples 9 and 10 illustrate the following result, extending Corollary 17 Its proof follows the exact same lines as the computations in those examples.

Corollary 11 If $\hat{\beta}$ is the closure of a braid of $n$ strands with $k_{+}$positive crossings and $k_{-}$negative crossings $\left(k_{+}>k_{-}\right)$, then $\tau(\hat{\beta}) \geq \frac{k_{+}-k_{-}-n+1}{2}$.

This corollary immediately gives the bound $g_{4}(\hat{\beta}) \geq \frac{k_{+}-k_{-}-n+1}{2}$, the SliceBennequin Inequality first proved by Rudolph in [12].

\section{Thurston-Bennequin Numbers}

Every knot has a polygonal diagram $D$ consisting of only vertical and horizontal segments, with each horizontal segment passing over the vertical. Corners in such a diagram are naturally labelled northeast, etc. As described in 13, the Thurston-Bennequin number of such a diagram, $\operatorname{tb}(D)$, is the difference of the writhe of the diagram and the number of northeast corners. Figure 4 illustrates a diagram $D$ of the trefoil knot with $\operatorname{tb}(D)=0$. (In defining the Thurston-Bennequin number, one usually considers knot diagrams in which all crossings are left handed with respect to the vertical direction and then takes the difference of the writhe and the number of right cusps. The definition here is simply obtained by "rotating" the standard definition by 45 degrees.) The Thurston-Bennequin number of a knot $K, \operatorname{TB}(K)$, is the maximum value of this quantity over all such diagrams for $K$. 


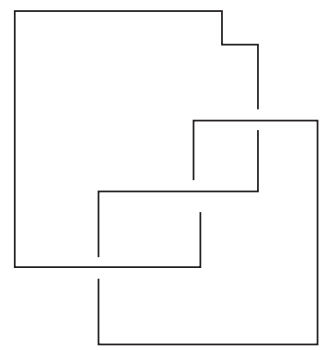

Figure 4

Theorem 12 If the Thurston-Bennequin number of a knot satisfies $T B(K) \geq$ 0 then all iterated untwisted (positive) Whitehead doubles of $K, W h_{n}(K)$, satisfy $\tau\left(W h_{n}(K)\right)=1$ and thus $g_{4}\left(W h_{n}(K)\right)=1$.

Proof Any polygonal diagram $D$ as above can be isotoped to a diagram $D^{\prime}$ with $\operatorname{tb}\left(D^{\prime}\right)=\operatorname{tb}(D)-1$; just add a new northeast corner without introducing any new crossings. (In Figure 4 an extra northeast corner was added to a diagram of the trefoil to illustrate this process.) Thus, we assume $\operatorname{tb}(D)=0$. As observed by Rudolph [13, this diagram quickly yields a placement of $K$ on the fiber $F$ of a torus knot for which the parallel copy $K^{\prime}$ of $K$ on $F$ has $\operatorname{link}\left(K, K^{\prime}\right)=0$. From this (eg, as in [13]) one sees there is also an unknotted curve $\alpha$ on $F$ meeting $K$ transversely in one point, with induced framing -1 . A neighborhood $G$ of $K \cup \alpha$ on $F$ is seen to be a genus 1 Seifert surface for the positive untwisted double of $K$, and hence by Theorem $4, T\left(W h_{1}(K)\right)=1$. (For this argument to work, one must in fact be a bit careful in the initial choice of $T_{p, q}$ and $F$; for some choices $K$ embeds, but not $\alpha$. Details can be found in the work of Rudolph.)

As shown in [1] and [13, a simple diagram reveals that $\operatorname{TB}\left(W h_{1}(K)\right) \geq 1$. Thus, this process can be iterated.

We close this section by noting that in independent work from that presented here, Olga Plamenevskaya [8] has described connections between the OzsváthSzabó theory, Thurston-Bennequin invariants, and their relationship to the 4-ball genus. 


\section{References}

[1] S Akbulut, R Matveyev, Exotic structures and adjunction inequality, Turkish J. Math. 21 (1997) 47-53

[2] A Kawamura, The unknotting numbers of $10_{139}$ and $10_{152}$ are 4, Osaka J. Math. 35 (1998) 539-546

[3] A Kawauchi, A survey of knot theory, Birkhäuser-Verlag, Basel (1996)

[4] P Kronheimer, T Mrowka, Gauge theory for embedded surfaces. I, Topology 32 (1993) 773-826

[5] C Livingston, Splitting the concordance group of algebraically slice knots, Geometry and Topology 7 (2003) 641-643

[6] B Owens, S Strle, Rational homology spheres and four-ball genus, preprint arXiv:math.GT/0308073

[7] P Ozsváth, Z Szabó, Knot Floer homology and the four-ball genus, Geometry and Topology 7 (2003) 615-639

[8] O Plamenevskaya, Bounds for Thurston-Bennequin number from Floer homology, arXiv:math.SG/0311090

[9] J A Rasmussen, Knot Floer homology, genus bounds, and mutation (2003), $\mathrm{PhD}$ thesis, Harvard University (2003)

[10] J A Rasmussen, Floer homologies of surgeries on two-bridge knots, Algebraic and Geometric Topology 2 (2003) 757-789

[11] D Rolfsen, Knots and links, Mathematics Lecture Series, 7. Publish or Perish, Inc., Houston, TX (1990)

[12] L Rudolph, Quasipositivity as an obstruction to sliceness, Bull. Amer. Math. Soc. 29 (1993) 51-59

[13] L Rudolph, An obstruction to sliceness via contact geometry and 'classical' gauge theory, Inv. Math. 119 (1995) 155-163

[14] L Rudolph, Quasipositive pretzels, Top. Appl. 115 (2001) 115-123

[15] T Shibuya, Local moves and 4-genus of knots, Memoirs of the Osaka Institute of Technology, Series A 45 (2000) 1-10

[16] T Tanaka, Unknotting numbers of quasipositive knots, Top. Appl. 88 (1998) 239-246 